\title{
OPEN Pre-infection plasma cytokines and chemokines as predictors of HIV disease progression
}

\author{
Samukelisiwe Ngcobo ${ }^{1,2}$, Refilwe P. Molatlhegi, ${ }^{1,2}$, Farzana Osman ${ }^{1}$, \\ Sinaye Ngcapu ${ }^{1,2}$, Natasha Samsunder ${ }^{1}$, Nigel J. Garrett ${ }^{1,6}$, Salim S. Abdool Karim ${ }^{1,4}$, \\ Quarraisha Abdool Karim ${ }^{1,4}$, Lyle R. McKinnon ${ }^{1,3,5,7}$ \& Aida Sivro ${ }^{1,2,7 凶}$
}

Previous studies have highlighted the role of pre-infection systemic inflammation on HIV acquisition risk, but the extent to which it predicts disease progression outcomes is less studied. Here we examined the relationship between pre-infection plasma cytokine expression and the rate of HIV disease progression in South African women who seroconverted during the CAPRISA 004 tenofovir gel trial. Bio-Plex 200 system was used to measure the expression of 47 cytokines/chemokines in 69 seroconvertors from the CAPRISA 004 trial. Cox proportional hazards regression analyses were used to measure associations between cytokine expression and CD4 decline prior to antiretroviral therapy initiation. Linear regression models were used to assess whether pre-infection cytokine expression were predictors of disease progression outcomes including peak and set-point viral load and CD4:CD8 ratio at less and greater than 180 days post infection. Several cytokines were associated with increased peak HIV viral load (including IL-16, SCGF $\beta$, MCP-3, IL-12p40, SCF, IFN $\alpha 2$ and IL-2). The strongest association with peak viral load was observed for SCGF $\beta$, which was also inversely associated with lowest CD4:CD8 ratio < 180 days post infection and faster CD4 decline below 500 cells/ $\mu$ l (adjusted HR 4.537, 95\% Cl 1.475-13.954; $p=0.008$ ) in multivariable analysis adjusting for age, study site, contraception, baseline HSV-2 status and trial arm allocation. Our results show that pre-infection systemic immune responses could play a role in HIV disease progression, especially in the early stages of infection.

Immune activation in response to HIV is critical in providing protective immunity to fight the infection, however those same responses can contribute to pathogenesis by providing the virus with increased supplies of activated target cells. Cytokine dysregulation and inflammation play major roles in HIV pathogenesis by contributing to $\mathrm{CD}^{+} \mathrm{T}$ cells depletion and increased viral load ${ }^{1-3}$. Immune activation was shown to be one of the major predictors of $\mathrm{CD}^{+} \mathrm{T}$ cell loss, independent of viral load ${ }^{4}$. The plasma cytokine storm during acute HIV infection predicts viral set-point and subsequent disease progression, with $66 \%$ of the variation in viral set-point 12 months post-infection predicted by plasma levels of 5 cytokines: interleukin (IL)-15, IL-7, IL-12p40, IL-12p70 and interferon gamma (IFNy) ${ }^{5,6}$. During chronic HIV infection, soluble plasma markers (such as IL-6, C-reactive protein and D-dimer) were shown to be predictive of comorbidities, disease progression and mortality in patients on and off antiretroviral therapy $(\mathrm{ART})^{7-10}$.

While several studies have shown that systemic immunity and increased inflammation increase the risk of HIV acquisition ${ }^{11-13}$, few have looked at the effect of pre-infection systemic inflammation on HIV disease progression ${ }^{14-16}$. High $\mathrm{CD} 4^{+} \mathrm{T}$ cell counts prior to infection were associated with significantly higher rate of $\mathrm{CD} 4^{+}$ T cell decline in the first 3 months of infection, likely related to increased HIV replication ${ }^{16}$. Understanding the impact of innate and adaptive immune function prior to HIV-1 infection on subsequent disease progression could contribute to the establishment of more optimized and personalized HIV treatment and prevention methods. In this study, we examined whether pre-infection plasma cytokine concentrations predicted the rate of HIV disease

${ }^{1}$ Centre for the AIDS Programme of Research in South Africa (CAPRISA), 719 Umbilo Road, Durban, South Africa. ${ }^{2}$ Department of Medical Microbiology, University of KwaZulu-Natal, Durban, South Africa. ${ }^{3}$ Department of Medical Microbiology, University of Manitoba, Winnipeg, Canada. ${ }^{4}$ Department of Epidemiology, Columbia University, New York, NY, USA. ${ }^{5}$ Department of Medical Microbiology, University of Nairobi, Nairobi, Kenya. ${ }^{6}$ Discipline of Public Health Medicine, School of Nursing and Public Health, University of KwaZulu-Natal, Durban, South Africa. ${ }^{7}$ These authors contributed equally: Lyle R. McKinnon and Aida Sivro. ${ }^{\boxplus}$ email: aida.sivro@ caprisa.org 
progression in the CAPRISA 004 cohort, a phase IIb randomized trial that evaluated the safety and effectiveness of $1 \%$ tenofovir gel for the prevention of HIV infection in South African women.

\section{Methods}

Study cohort. This study included women who HIV seroconverted while participating in CAPRISA 004, a phase IIb, randomised, double-blinded, placebo-controlled study of $1 \%$ tenofovir gel: "Safety and Effectiveness Study of a Candidate Vaginal Microbicide for Prevention of HIV" (NCT00441298) ${ }^{17}$. CAPRISA 004 enrolled sexually active, HIV uninfected females, aged between 18-40 years from rural (Vulindlela Research Clinic) and urban clinics (eThekwini Research Clinic) in KwaZulu Natal, South Africa from May 2007 to January 2009. Once seroconversion was confirmed, women were then followed up in the CAPRISA 002 Acute Infection cohort study $^{18}$. This study was approved by the University of KwaZulu Natal Biomedical Research Ethics Committee (BREC/00001105/2020), and all participants provided the informed written consent prior to enrollment. All methods were carried out in accordance with relevant guidelines and regulations.

Sample collection and processing. Peripheral blood was collected in acid citrate dextrose (ACD) tubes. Following centrifugation $\left(10^{\prime}, 1600 \mathrm{rpm}\right)$ plasma was separated and cryopreserved at $-80^{\circ} \mathrm{C}$ for further analysis.

Soluble biomarker analysis. Cytokine/chemokine levels were measured using the Bio-Plex cytokine assays Group 1 (27 Plex) and Group II (21 Plex). Human cytokine 27-plex Assay (\#M500KCAF0Y, BIO-RAD) included the following cytokines: IL-1 $\beta$, IL-1ra, IL-2, IL-4, IL-5, IL-6, IL-7, IL-8, IL-9, IL-10, IL-12p70, IL-13, IL-15, IL-17 $\alpha$, Eotaxin, Basic FGF, G-CSF, GM-CSF, IFN-y, IP-10, MCP-1,MIP- $1 \alpha$, MIP-1 $\beta$, PDGF- $\beta \beta$, RANTES, TNF- $\alpha$. Human 21-plex Assay (\#MF0005KMII, BIO-RAD) included: IFN- $\alpha 2$, IL-1 $\alpha$, IL-2Ra, IL-3, IL-12p40, IL-16, CTACK, GRO $\alpha$, HGF, LIF, MCP-3, M-CSF, MIF, MIG, $\beta$-NGF, SCF, SCGF $\beta$, SDF- $1 \alpha$, TNF- $\beta$, and TRAIL. All assays were performed following manufacturer's instructions. Samples with values below the lower detection limit were assigned the value half the lower limit of quantification, LLOQ/2.

Statistical analysis. All cytokine concentrations were $\log _{10}$ transformed. Cytokines detectable at less than $60 \%$ frequency were analysed as binary variables (IL-1 $\alpha$ and IL-2), and the remainder were analysed as continuous variables. Disease progression was evaluated using several readouts including: HIV viral load (VL), measured both as peak (highest value in the first 180 days of infection) and set point (average value in measurements made after 180 days of infection prior to ART initiation); and CD4:CD8 ratio, measured both as lowest CD4:CD8 ratio within the first 180 days post infection and the mean CD4:CD8 ratio $>180$ days (average value in measurements made after 180 days of infection prior to ART initiation). Linear regression was used to assess the impact of each cytokine on VL and CD4:CD8 ratio in bivariate and multivariable models adjusting for age, contraceptive use, HSV-2 at baseline, study site and study arm at randomization. The rates of CD4 decline were compared in bivariate and multivariable Cox regression models with the endpoint defined as two consecutive CD4 counts below 500 cells/ $\mu$ l. Analysis excluded the CD4 counts during the first 180 days post infection due to transient CD4 decreases during acute infection, as previously described ${ }^{19}$. Multivariable analysis with and without the $\log \mathrm{VL}$ as a predictor variable is reported for the CD4 decline analysis. Statistical significance was indicated as $p$ value $\leq 0.05$. Multiple comparisons adjustment was done using false discovery rate (FDR) method of Benjamini and Hochberg with FDR $(\mathrm{Q})=10 \%$. All analyses were performed using SPSS v27 and GraphPad Prism Version 7.

\section{Results}

Study participants. We measured the expression of plasma cytokines from women who became HIV infected at three months after enrolment in the CAPRISA 004 study. A total of 69 women were sampled and 60 included in the final analysis. Plasma samples were obtained at a median of 330 days prior to infection (IQR 211-493). The median age was 22 (IQR 20-25), and the baseline HSV-2 seroprevalence was 63.2\%. Most participants (73.9\%) were using Depo-Provera (DMPA) as their preferred method of contraception. The demographic, behavioural and clinical variables are shown in Table 1.

Pre-infection plasma cytokines/chemokines and viral load. We performed bivariate and multivariable linear regression models to assess associations between pre-infection cytokines and HIV viral load. In bivariate analyses IL-16, serum stem cell growth factor beta (SCGF $\beta$ ), monocyte chemotactic protein-3 (MCP-3), IL12 p40 and stem cell factor (SCF) concentrations were associated with increased peak viral load (Table 2). Similar results were obtained for these cytokines in models adjusted for contraceptive use, age, HSV-2 at baseline, study site and study arm [IL-16, adjusted(a) $\beta=2.065,95 \%$ CI 0.786, 3.344, $p=0.002$; SCGF $\beta$, a $\beta=1.345,95 \%$ CI 0.536, 2.154, $p=0.002$; MCP-3, $\mathrm{a} \beta=1.101,95 \%$ CI $0.255,1.946, p=0.012$; IL-12p40, $\beta=0.643,95 \%$ CI $0.126,1.160$, $p=0.016$; SCF, $\mathrm{a} \beta=0.989,95 \%$ CI $0.019,1.959, p=0.046$; Table 2, Fig. 1]. Additionally, IFNa 2 and IL-2 were significantly associated with peak viral load in multivariable analysis (IFNa2, a $\beta=1.277,95 \%$ CI, 0.088, 2.466, $p=0.036$; IL-2, a $\beta=0.835,95 \%$ CI 0.011, 1.658, $p=0.047$; Table 2, Fig. 1). We observed no significant association between pre-infection cytokines and set-point viral load (Sup. Table 2). It is likely that through their chemotactic and pro-inflammatory properties, persistent upregulation of the identified markers can lead to increased number of activated $\mathrm{CD} 4^{+} \mathrm{T}$ cells thereby fuelling early HIV replication.

Pre-infection plasma cytokines/chemokines and CD4:CD8 ratio post-infection. Next we used linear regression models to evaluate if pre-infection cytokine expression was associated with lowest CD4:CD8 


\begin{tabular}{|c|c|}
\hline Variables & Cases $(n=69)$ \\
\hline Age, years [median (IQR)] & $22(20-25)$ \\
\hline Sex acts in the last 30 days [median (IQR)] & $6(3-10)$ \\
\hline Oldest sex partner, years [median (IQR)] & $25(23-28)$ \\
\hline \multicolumn{2}{|l|}{ Living with regular partner } \\
\hline No & $62(89.90 \%)$ \\
\hline Yes & $7(10.10 \%)$ \\
\hline \multicolumn{2}{|l|}{ Highest level of education } \\
\hline Primary school not complete & $3(4.3 \%)$ \\
\hline Primary Schooling complete & $37(53.6 \%)$ \\
\hline High school complete & $28(40.6)$ \\
\hline Tertiary education complete & $1(1.4 \%)$ \\
\hline \multicolumn{2}{|l|}{ Contraception } \\
\hline Depo-provera & $51(73.90 \%)$ \\
\hline Hysterectomy & 0 \\
\hline Nur-Isterate & $13(18.80 \%)$ \\
\hline Oral & $5(7.20 \%)$ \\
\hline Tubal ligation & 0 \\
\hline \multicolumn{2}{|l|}{ Marital status } \\
\hline Casual partner(s) & $2(2.90 \%)$ \\
\hline Married & $2(2.90 \%)$ \\
\hline Married and have casual partners & 0 \\
\hline Stable partner(s) & $61(88.40 \%)$ \\
\hline Stable and casual partners & $4(5.80 \%)$ \\
\hline \multicolumn{2}{|l|}{ Treatment arm (CAPRISA 004) } \\
\hline Placebo & $42(60.90 \%)$ \\
\hline Tenofovir & $27(39.10 \%)$ \\
\hline \multicolumn{2}{|l|}{ Baseline HSV-2 } \\
\hline No & $25(36.80 \%)$ \\
\hline Yes & $43(63.20 \%)$ \\
\hline
\end{tabular}

Table 1. Study cohort characteristics.

\begin{tabular}{|c|c|c|c|c|c|c|c|c|}
\hline \multirow[b]{3}{*}{ Variables } & \multicolumn{4}{|l|}{ Bivariate } & \multicolumn{4}{|l|}{ Multivariable } \\
\hline & \multirow[b]{2}{*}{ B-coefficient } & \multicolumn{2}{|l|}{$95 \% \mathrm{CI}$} & \multirow[b]{2}{*}{$p$ Value } & \multirow[b]{2}{*}{ a $\beta$-coefficient } & \multicolumn{2}{|l|}{$95 \%$ CI } & \multirow[b]{2}{*}{$p$ Value } \\
\hline & & Lower & Upper & & & Lower & Upper & \\
\hline Log IL-16 & 1.939 & 0.732 & 3.146 & $0.002^{\star}$ & 2.065 & 0.786 & 3.344 & $0.002^{\star}$ \\
\hline $\log \mathrm{SCGF} \beta$ & 1.310 & 0.517 & 2.104 & $0.002^{*}$ & 1.345 & 0.536 & 2.154 & $0.002^{\star}$ \\
\hline Log MCP-3 & 0.858 & 0.063 & 1.653 & 0.035 & 1.101 & 0.255 & 1.946 & 0.012 \\
\hline Log IL-12p40 & 0.500 & 0.031 & 0.969 & 0.037 & 0.643 & 0.126 & 1.160 & 0.016 \\
\hline Log SCF & 0.950 & 0.039 & 1.861 & 0.041 & 0.989 & 0.019 & 1.959 & 0.046 \\
\hline $\log$ IFNa2 & 0.975 & -0.145 & 2.096 & 0.087 & 1.277 & 0.088 & 2.466 & 0.036 \\
\hline Log IL-2 & 0.679 & -0.096 & 1.454 & 0.085 & 0.835 & 0.011 & 1.658 & 0.047 \\
\hline
\end{tabular}

Table 2. Bivariate and multivariable linear regression analysis on the effect of pre-infection plasma cytokines on peak viral load (*only statistically significant and trending cytokines/chemokines shown, for rest of the data see Sup. Table 1). Significant values are in bold. Dependent variable: Log10 viral load; both models included study arm; multivariable analysis adjusted for contraception, age, study site, HSV-2 at baseline and study arm. $p$ Values that passed the FDR correction are indicated with $\mathrm{a}^{*}$.

ratio $<180$ days post infection. Six cytokines were negatively associated with lowest CD4:CD8 ratio in the bivariate model: IL-10, MCP-1, SCGF $\beta$, IL-1 ra, IL-6 and IL-2 (Table 3). Similar results were obtained following multivariable analysis: IL-10: $\mathrm{a} \beta=-0.216,95 \%$ CI $(-0.369,-0.062), p=0.007 ; \mathrm{MCP}-1: \mathrm{a} \beta=-0.273 ; 95 \%$ CI $(-0.530,-0.017), p=0.037$; SCGF $\beta: \mathrm{a} \beta=-0.344,95 \%$ CI $(-0.644,-0.043), p=0.026$; IL-1 $\mathrm{r} \alpha \mathrm{a} \beta=-0.551,95 \%$ CI $(-1.021,-0.082), p=0.022$; IL-6: $\mathrm{a} \beta=-0.361,95 \%$ CI $(-0.662,-0.059), p=0.020$; IL-2: $\mathrm{a} \beta=-0.347,95 \%$ CI $(-0.635,-0.060), p=0.019$; Table 3, Fig. 2 A. As SCGF $\beta$ was also associated with peak viral load, its effect on decreased CD4:CD8 ratio is likely mediated through increase in HIV replication and associated CD4 ${ }^{+} \mathrm{T}$ cell 

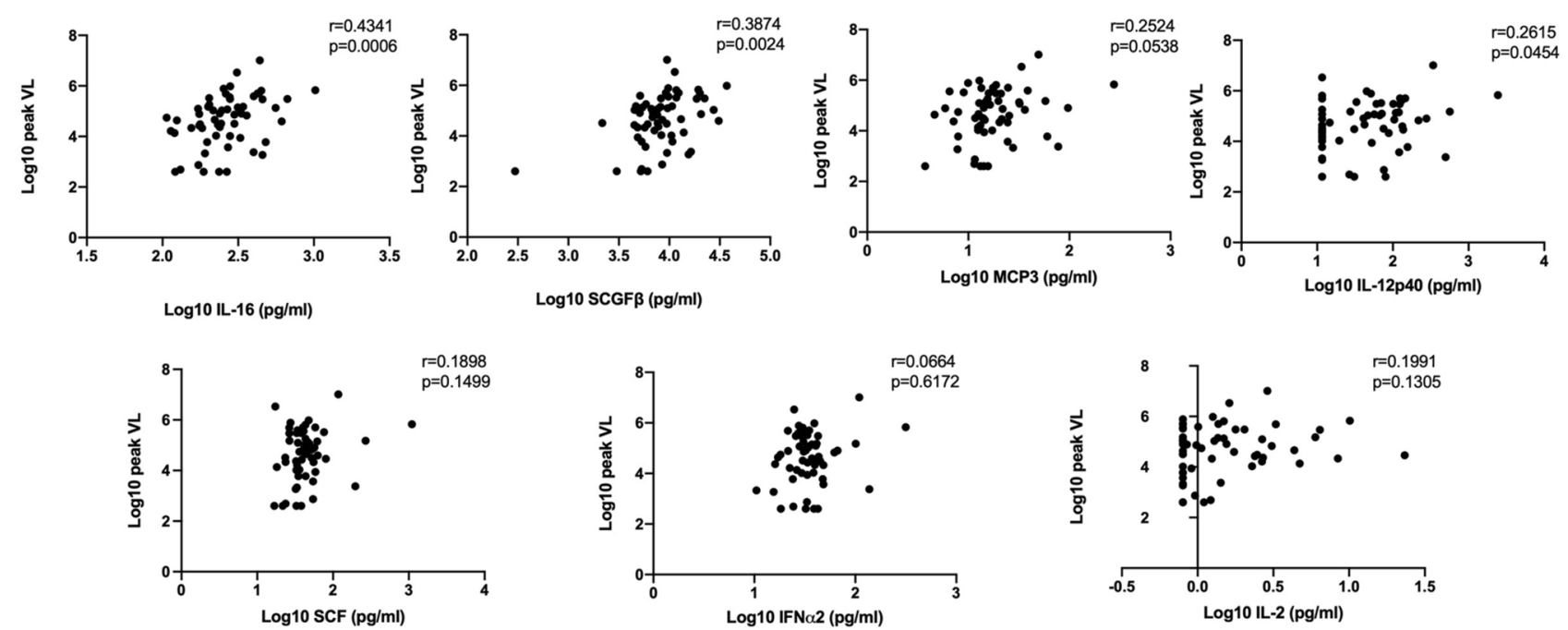

Figure 1. Correlation between plasma cytokines and peak VL $(n=60)$. Only significantly affected cytokines in multivariable linear regression analysis are shown in the figure. The $\mathrm{r}$ and $p$ values depicted in the graphs are the result of the Spearman correlation analysis.

\begin{tabular}{|c|c|c|c|c|c|c|c|c|}
\hline \multirow[b]{3}{*}{ Variables } & \multicolumn{4}{|l|}{ Bivariate } & \multicolumn{4}{|l|}{ Multivariable } \\
\hline & \multirow[b]{2}{*}{$\beta$-coefficient } & \multicolumn{2}{|l|}{ 95\% CI } & \multirow[b]{2}{*}{$p$ Value } & \multirow[b]{2}{*}{$\mathbf{a} \beta$-coefficient } & \multicolumn{2}{|l|}{$95 \% \mathrm{CI}$} & \multirow[b]{2}{*}{$p$ Value } \\
\hline & & \begin{tabular}{|l|} 
Lower \\
\end{tabular} & Upper & & & \begin{tabular}{|l|} 
Lower \\
\end{tabular} & Upper & \\
\hline Log IL-10 & -0.206 & -0.353 & -0.060 & 0.007 & -0.216 & -0.369 & -0.062 & 0.007 \\
\hline Log MCP-1 & -0.284 & -0.533 & -0.035 & 0.026 & -0.273 & -0.530 & -0.017 & 0.037 \\
\hline $\log \mathrm{SCGF} \beta$ & -0.373 & -0.663 & -0.083 & 0.013 & -0.344 & -0.644 & -0.043 & 0.026 \\
\hline Log IL-1ra & -0.571 & -1.027 & -1.114 & 0.015 & -0.551 & -1.021 & -0.082 & 0.022 \\
\hline Log IL-6 & -0.351 & -0.645 & -0.057 & 0.020 & -0.361 & -0.662 & -0.059 & 0.020 \\
\hline Log IL-2 & -0.329 & -0.597 & -0.061 & 0.017 & -0.347 & -0.635 & -0.060 & 0.019 \\
\hline
\end{tabular}

Table 3. Bivariate and multivariable linear regression analysis on the effect of pre-infection plasma cytokines on minimum $\mathrm{CD} 4: \mathrm{CD} 8$ ratio $<180$ post infection ( ${ }^{*}$ only statistically significant and trending cytokines/ chemokines shown, for rest of the data see Sup. Table 3). Significant values are in bold. Dependent variable: CD4:CD8 ratio; both models included study arm; multivariable analysis adjusted for contraception, age, study site, HSV-2 at baseline and study arm.

death, while the effect of the other cytokines could be mediated through immune activation/exhaustion. Fewer associations with CD4:CD8 ratio > 180 days post infection were observed, with IL-10 and stromal cell-derived factor-1 alpha (SDF-1 $\alpha$ ) being significant in bivariate analysis (Table 4). IL-10 remained significantly associated after adjusting for contraception, age, study site, study arm, age and HSV-2 serostatus at baseline [a $\beta=-0.153$, $95 \%$ CI $(-0.282,-0.024), p=0.021]$ Table 4, Fig. 2B. Additionally, increased IL-12p70 was significantly associated with lower mean CD4:CD8 ratios $>180$ in the multivariable analysis $[\mathrm{a} \beta=-0.202,95 \% \mathrm{CI}(-0.376,-0.029)$, $p=0.023]$ Table 4, Fig. 2B.

Pre-infection plasma cytokine expression and CD4 decline. We next performed bivariate and multivariable Cox regression analyses of pre-infection plasma cytokines and time to CD4 decline below 500 cells/ $\mu \mathrm{l}$ prior to ART initiation. Pre-infection plasma SCGF $\beta(\mathrm{HR}=3.71,95 \%$ CI $1.28-10.77, p=0.016)$ and TNF $\beta$ ( $\mathrm{HR}=2.24,95 \% \mathrm{CI} 1.13-4.45, p=0.022)$ levels significantly predicted faster CD4 loss in the bivariate analysis (Table 5). Additionally, there was a trend for IL-16 (HR=4.546, 95\% CI 0.919-22.484, $p=0.063$, Table 5). In the multivariable model adjusting for contraception, age, study site, study arm, VL and HSV-2 at baseline IL-2 $(\mathrm{aHR}=0.318,95 \% \mathrm{CI} 0.109-0.926, p=0.036)$, growth-regulated oncogene alpha (GROa $)(\mathrm{aHR}=0.13,95 \% \mathrm{CI}$ $0.018-0.965, p=0.046)$, IFNa2 $(\mathrm{aHR}=0.118,95 \%$ CI $0.016-0.847, p=0.034)$ and SDF- $1 \alpha(\mathrm{aHR}=0.087,95 \%$ CI $0.009-0.853, p=0.036$ ) correlated with slower CD4 decline (Sup. Table 5). This model corrected for HIV viral load indicating that these cytokines likely affect CD4 decline in viral load-independent mechanisms, most likely due to increased $\mathrm{T}$ cell function and upregulation of anti-viral immune responses. We next ran a multivariable model excluding viral load, as seen in the bivariate model, SCGF $\beta$ (aHR $=4.537,95 \%$ CI $1.475-13.954$, $p=0.008)$ and TNF $\beta(\mathrm{aHR}=2.318,95 \%$ CI 1.133-4.741, $p=0.021$ (Table 5) were associated with faster CD4 decline suggesting that this effect may be due to increases in viral replication, in addition to increased immune 
A
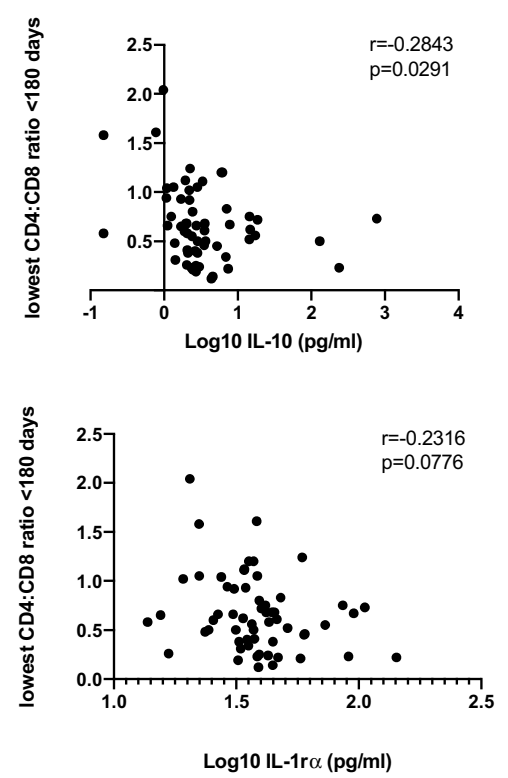

B

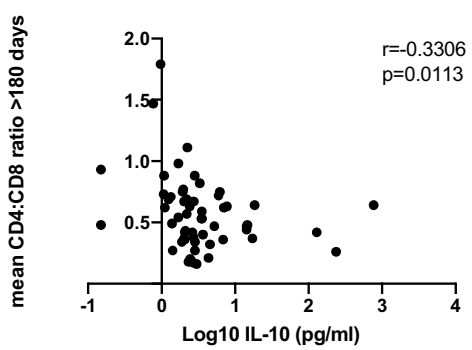

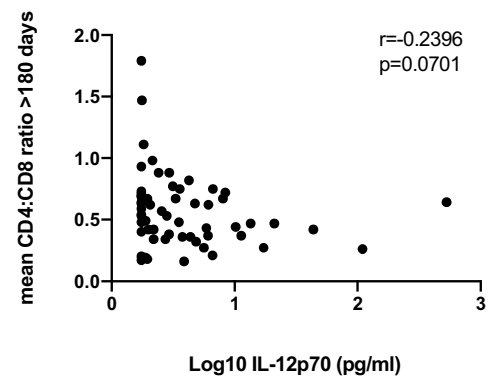

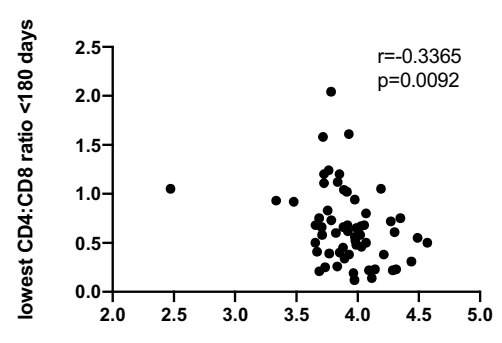

$\log 10$ SCGF $\beta(\mathrm{pg} / \mathrm{ml})$
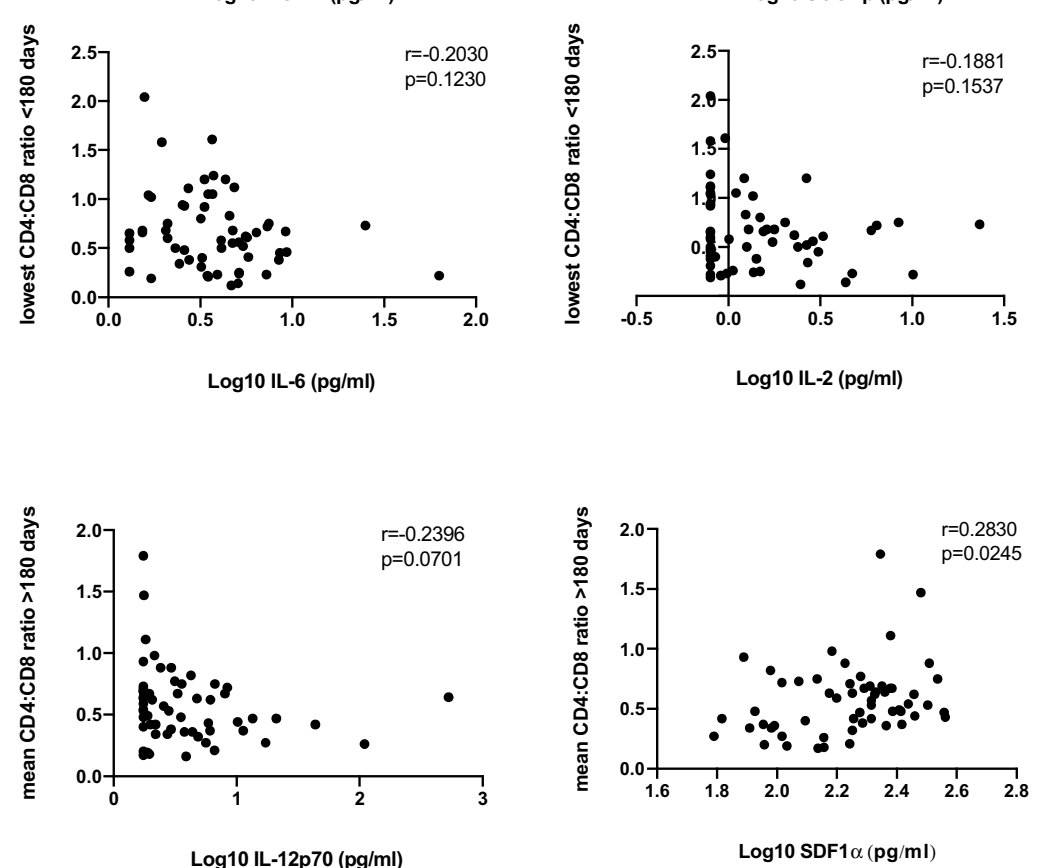

Figure 2. Correlation between plasma cytokines and lowest CD4:CD8 ratio $<180$ days post infection (A) and mean CD4:CD8 ratio $>180$ days post infection $(B)(n=60)$. Only significantly affected cytokines in multivariable linear regression analysis are shown in the figure. The $r$ and $p$ values depicted in the graphs are the result of the Spearman correlation analysis.

\begin{tabular}{|c|c|c|c|c|c|c|c|c|}
\hline \multirow[b]{3}{*}{ Variables } & \multicolumn{4}{|l|}{ Bivariate } & \multicolumn{4}{|l|}{ Multivariable } \\
\hline & \multirow[b]{2}{*}{$\beta$-coefficient } & \multicolumn{2}{|l|}{$95 \%$ CI } & \multirow[b]{2}{*}{$p$ Value } & \multirow[b]{2}{*}{ a $\beta$-coefficient } & \multicolumn{2}{|l|}{$95 \%$ CI } & \multirow[b]{2}{*}{$p$ Value } \\
\hline & & Lower & Upper & & & Lower & Upper & \\
\hline Log IL-10 & -0.135 & -0.262 & -0.008 & 0.038 & -0.153 & -0.282 & -0.024 & 0.021 \\
\hline Log IL-12p70 & -0.130 & -0.295 & 0.035 & 0.120 & -0.202 & -0.376 & -0.029 & 0.023 \\
\hline Log SDF-1a & 0.430 & 0.047 & 0.814 & 0.029 & 0.248 & -0.297 & 0.793 & 0.365 \\
\hline
\end{tabular}

Table 4. Bivariate and multivariable linear regression analysis on the effect of pre-infection plasma cytokines on mean CD4:CD8 ratio $>180$ post infection $\left({ }^{*}\right.$ only significant and trending cytokines/chemokines shown, for rest of the data see Sup. Table 4). Significant values are in bold. Dependent variable: CD4:CD8 ratio; both models included study arm; multivariable analysis adjusted for contraception, age, study site, HSV-2 at baseline and study arm.

activation. We additionally observed a trend for IL-16 ( $\mathrm{aHR}=4.595,95 \%$ CI 0.907-23.270, $p=0.065)$ and MIP1 $\beta$ $(\mathrm{aHR}=4.079,95 \%$ CI $0.957-17.391, p=0.057)$ being associated with faster CD4 decline.

\section{Discussion}

Systemic inflammation is considered to be a driving force underlying $\mathrm{CD} 4^{+} \mathrm{T}$ cell depletion and immune dysfunction during HIV disease progression, and it is known to persist in ARV-treated individuals with suppressed viral load ${ }^{20}$. Numerous studies have investigated the effects of systemic inflammation during the acute and chronic infection; however, few have assessed the impact of pre-infection immune status on disease progression. Pre-infection levels of cytokines likely reflect the immunological status of the individual and could therefore be important in assessing the risk of early stage disease progression. Here we characterised the impact of 


\begin{tabular}{|l|l|l|l|l|l|l|}
\hline Variables & HR $(\mathbf{9 5} \%$ CI $)$ & $\boldsymbol{p}$ Value & aHR ${ }^{*} \mathbf{9 5} \%$ CI $)$ & $\boldsymbol{p}$ Value & aHR $(-\mathbf{V L})^{*}(\mathbf{9 5} \%$ CI $)$ & $\boldsymbol{p}$ Value \\
\hline $\log$ SCGF $\beta$ & $3.712(1.279-10.771)$ & $\mathbf{0 . 0 1 6}$ & $2.738(0.729-10.290)$ & 0.136 & $4.537(1.475-13.954)$ & $\mathbf{0 . 0 0 8}$ \\
\hline $\log$ TNF $\beta$ & $2.236(1.125-4.445)$ & $\mathbf{0 . 0 2 2}$ & $1.745(0.813-3.746)$ & 0.153 & $2.318(1.133-4.741)$ & $\mathbf{0 . 0 0 2}{ }^{*}$ \\
\hline $\log$ IL-16 & $4.546(0.919-22.484)$ & 0.063 & $0.988(0.154-6.326$ & 0.990 & $4.595(0.907-23.270)$ & 0.065 \\
\hline $\log$ IL-2 & $0.936(0.418-2.094)$ & 0.871 & $0.318(0.109-0.926)$ & $\mathbf{0 . 0 3 6}$ & $0.905(0.368-2.225)$ & 0.828 \\
\hline $\log$ GRO $\alpha$ & $0.387(0.081-1.846)$ & 0.234 & $0.131(0.018-0.965)$ & $\mathbf{0 . 0 4 6}$ & $0.416(0.084-2.051)$ & 0.281 \\
\hline $\log$ IFNa2 & $1.208(0.232-6.287)$ & 0.822 & $0.118(0.016-0.847)$ & $\mathbf{0 . 0 3 4}$ & $1.663(0.277-9.970)$ & 0.578 \\
\hline $\log$ SDF-1 $\alpha$ & $0.666(0.159-2.792)$ & 0.578 & $0.087(0.009-0.853)$ & $\mathbf{0 . 0 3 6}$ & $1.082(0.145-8.076)$ & 0.939 \\
\hline $\log$ IL-10 & $1.109(0.743-1.656)$ & 0.613 & $0.592(0.349-1.005)$ & 0.052 & $1.046(0.657-1.666)$ & 0.850 \\
\hline $\log$ IL-3 & $0.866(0.248-3.022)$ & 0.822 & $0.268(0.069-1.039)$ & 0.057 & $1.083(0.269-4.359)$ & 0.910 \\
\hline $\log$ TRAIL & $0.728(0.303-1.751)$ & 0.479 & $0.446(0.196-1.015)$ & 0.054 & $0.641(0.247-1.660)$ & 0.360 \\
\hline $\log$ MIP-1 $\beta$ & $2.369(0.630-8.911)$ & 0.202 & $3.882(0.675-22.339)$ & 0.129 & $4.079(0.957-17.391)$ & 0.057 \\
\hline
\end{tabular}

Table 5. Bivariable and multivariable survival analysis of the effect of pre-infection plasma cytokines on CD4 decline $<500 \mu \mathrm{l}$ ( ${ }^{*}$ only statistically significant and trending cytokines/chemokines shown, for rest of the data see Sup. Table 5). Significant values are in bold. Study arm was included in all models; multivariable analysis adjusted for contraception, age, study site, HSV-2 at baseline study arm, + / - VL. $p$ Values that passed the FDR correction are indicated with $\mathrm{a}^{*}$.

pre-infection plasma cytokines on the markers of HIV disease progression including viral load, CD4:CD8 ratio and CD4 decline.

We found that expression of five cytokines, IL-16, SCGF $\beta$, MCP-3, IL-12p40, and SCF had an impact on the peak viral load following bivariate and multivariable linear regression analyses. The observed effect was strongest for IL-16 and SCGF $\beta$. IL-16, originally named as lymphocyte chemoattractant factor (LCF), is produced by a range of cell types including activated $\mathrm{CD} 8^{+} \mathrm{T}$ cells, macrophages and dendritic, and it is a potent chemoattractant for all peripheral immune cells expressing CD4 receptor ${ }^{21}$. IL-16 was shown to prevent HIV replication ex vivo, with the inhibitory effect likely mediated at the level of transcriptional regulation ${ }^{21-25}$. While IL-16 can likely contribute to hindrance of HIV replication at the site of exposure, once infection is established increased systemic levels of IL-16, can contribute to increased $\mathrm{CD} 4^{+} \mathrm{T}$ cell recruitment during acute infection thereby increasing HIV replication and subsequent disease progression. SCGF $\beta$ is a growth factor for hematopoietic progenitor cells (HPCs) and has chemotactic properties ${ }^{26}$. While there is a lack of data on the role of the SCGF $\beta$ in inflammation and HIV infection, elevated plasma levels of SCGF $\beta$ could potentially induce proliferation and activation of HIV target cells, thereby contributing to the increased viral load during early infection. During early infection, MCP-3 and IL-12p40, through their pro-inflammatory properties and as a cause and consequence of macrophage/dendritic cell recruitment, can lead to increased HIV replication ${ }^{27-31}$. We also found IFN- $\alpha 2$ to be significantly associated with peak-viral load. Continuous production of IFN- $\alpha$ has been associated with rapid HIV-disease progression, based on the likely effect of IFN- $a$ to induce T cell differentiation and activation ${ }^{32}$. It has also been shown that transmitted founder viruses are most likely to replicate and spread in the presence of IFN- $\alpha^{33}$. SCF and IL-2 promote differentiation and proliferation of HIV target cells ${ }^{34}$ and could contribute to increase in the availability of cells susceptible to HIV infection, thereby resulting in increased viral replication. We observed no significant associations between pre-infection soluble cytokine/chemokine expression and set point VL, suggesting that pre-infection immune status likely has a greater impact on early stages of HIV infection. HIV viral load is likely determined by other factors including CD8 responses after infection is established ${ }^{35}$.

In addition to viral load, CD4:CD8 ratio is another commonly used marker of HIV disease progression and HIV-linked immune dysregulation ${ }^{36}$. HIV is associated with the reduction of CD4 ${ }^{+} \mathrm{T}$ cells and the increase of activated cytotoxic $\mathrm{T}$ lymphocytes specific to HIV ${ }^{37}$. Here we found that increased plasma concentrations of IL-10, MCP-1, IL-1ra, IL-2, IL-6 and SCGF $\beta$ had a negative impact on CD4:CD8 ratio during acute infection ( $<180$ days post infection), as evaluated by linear regression model. IL-10 is part of the broad "cytokine storm" in acute HIV infection and limits the magnitude and the functional capacity of effector CD $4^{+} \mathrm{T}$ cells ${ }^{38-40}$. IL-1ra is an inflammatory cytokine that is rapidly produced by numerous cells in response to infection and modulates IL-1 activity ${ }^{41}$. Increased IL-1ra and IL10 expression prior to infection can compromise early immune response to HIV and result in reduced CD4:CD8 ratio. MCP1, SCGF $\beta$ and IL-6 effect is likely mediated through its chemotactic and pro-inflammatory properties ${ }^{26,42}$ and $\mathrm{CD}^{+} \mathrm{T}$ cell polarization towards a Th2 phenotype ${ }^{43}$. Furthermore, IL-10, IL-12p70 and SDF-1 $\alpha$ were found to be significantly associated with lower CD4:CD8 ratios during chronic phase (>180 days post infection). Since IL-10 is involved in lessening the effector CD $4^{+} \mathrm{T}$ cell responses, increased pre-infection levels of this cytokine can potentially downregulate immune responses responsible for combating HIV. Increased pre-infection inflammation likely has a more profound effect during acute infection through HIV target cell recruitment and activation, while effects on later stages of infection are mediated through impairment and downregulation of host immune responses.

We also examined the effect of pre-infection plasma cytokine levels on CD4 ${ }^{+} \mathrm{T}$ cell decline below $500 \mu \mathrm{l}$ prior to ART initiation. Increased understanding of the causes of CD4 decline in HIV patients is important for clinical management of the disease ${ }^{44}$. In our study, 2 of the pre-infection plasma cytokines (SCGF $\beta$ and TNF $\beta$ ) significantly predicted faster $\mathrm{CD} 4{ }^{+} \mathrm{T}$ loss. Cytokines that were associated with slower CD4 decline in the multivariable model adjusting for VL included IL-2, GROa, IFNa2, SDF-1 $\alpha$. Since VL is one of the main drivers of CD4 
decline during HIV infection, in order to determine if the effect of cytokines identified in the bivariate analysis was mainly driven by VL, we re-ran the multivariable model excluding correction for VL measure. In the multivariable model excluding VL, following cytokines were significantly associated with CD4 decline: MIP1 $\beta$, IL-16, SCGF $\beta$ and TNF $\beta$ indicating that their effect on CD4 decline is at least partly mediated through HIV replication and the associated VL changes. This is supported by the results looking at the peak VL, where both IL-16 and SCGF $\beta$ were associated with increased peak VL. The effect of pre-infection IL-2, GRO $\alpha$, IFN $\alpha 2$, and SDF1- $\alpha$ on CD4 decline is likely to be independent of VL and HIV replication and instead may be reflective of pre-infection immune state that is favourable in the context of HIV disease progression once the person is infected.

Our study has several limitations. Our findings are specific to young women in South Africa and should be validated in other settings. We cannot exclude the effect of other unmeasured co-infections on the inflammatory signatures in the studied cohort. Additional limitation of this study includes the fact that the cytokine measurements were not made at the last HIV-negative visit, closest to the infection time. However, despite this, our study shows that pre-infection systemic cytokine/chemokine expression seems to play a significant role in determining the rate of disease progression. Once a person is infected, level of existing systemic inflammation can determine the amount of activated $\mathrm{CD} 4^{+} \mathrm{T}$ cells that are available to the virus; increased levels of activated $\mathrm{CD} 4^{+} \mathrm{T}$ cells can result in increased peak viral load and a more profound drop in $\mathrm{CD} 4^{+} \mathrm{T}$ cell numbers ultimately leading to worsened disease progression outcome.

Received: 17 August 2021; Accepted: 2 February 2022

Published online: 14 February 2022

\section{References}

1. Goujard, C. et al. CD4 cell count and HIV DNA level are independent predictors of disease progression after primary HIV type 1 infection in untreated patients. Clin. Infect. Dis. https://doi.org/10.1086/500213 (2006).

2. Shebl, F. M., Yu, K., Landgren, O., Goedert, J. J. \& Rabkin, C. S. Increased levels of circulating cytokines with HIV-related immunosuppression. AIDS Res. Hum. Retrovir. https://doi.org/10.1089/aid.2011.0144 (2012).

3. Leeansyah, E., Malone, D. F. G., Anthony, D. D. \& Sandberg, J. K. Soluble biomarkers of HIV transmission, disease progression and comorbidities. Curr. Opin. HIV AIDS https://doi.org/10.1097/COH.0b013e32835c7134 (2013).

4. Deeks, S. G. et al. Immune activation set point during early HIV infection predicts subsequent CD4+ T-cell changes independent of viral load. Blood https://doi.org/10.1182/blood-2003-09-3333 (2004).

5. Jiao, Y. et al. Plasma IP-10 is associated with rapid disease progression in early HIV-1 infection. Viral Immunol. https://doi.org/ 10.1089/vim.2012.0011 (2012).

6. Roberts, L. et al. Plasma cytokine levels during acute HIV-1 infection predict HIV disease progression. AIDS https://doi.org/10. 1097/QAD.0b013e3283367836 (2010).

7. Kuller, L. H. et al. Inflammatory and coagulation biomarkers and mortality in patients with HIV infection. PLoS Med. https://doi. org/10.1371/journal.pmed.0050203 (2008).

8. Boulware, D. R. et al. Higher levels of CRP, D-dimer, IL-6, and hyaluronic acid before initiation of antiretroviral therapy (ART) are associated with increased risk of AIDS or death. J. Infect. Dis. https://doi.org/10.1093/infdis/jir134 (2011).

9. Hileman, C. O. et al. Elevated D-dimer is independently associated with endothelial dysfunction: A cross-sectional study in HIVinfected adults on antiretroviral therapy. Antivir. Ther. https://doi.org/10.3851/IMP2297 (2012).

10. Tien, P. C. et al. Inflammation and mortality in HIV-infected adults: Analysis of the FRAM study cohort. J. Acquir. Immune Defic. Syndr. https://doi.org/10.1097/QAI.0b013e3181e66216 (2010).

11. Kahle, E. M. et al. Plasma cytokine levels and risk of HIV type 1 (HIV-1) transmission and acquisition: A nested case-control study among HIV-1-serodiscordant couples. J. Infect. Dis. https://doi.org/10.1093/infdis/jiu621 (2015).

12. Liebenberg, L. J. P. et al. Genital-systemic chemokine gradients and the risk of HIV acquisition in women. J. Acquir. Immune Defic. Syndr. https://doi.org/10.1097/QAI.0000000000001218 (2017).

13. Naranbhai, V. et al. Innate immune activation enhances HIV acquisition in women, diminishing the effectiveness of tenofovir microbicide gel. J. Infect. Dis. https://doi.org/10.1093/infdis/jis465 (2012).

14. Sivro, A. et al. Integrin $\alpha 4 \beta 7$ expression on peripheral blood CD $4+\mathrm{T}$ cells predicts HIV acquisition and disease progression outcomes. Sci. Transl. Med. https://doi.org/10.1126/scitranslmed.aam6354 (2018).

15. Hazenberg, M. D. et al. Persistent immune activation in HIV-1 infection is associated with progression to AIDS. AIDS https://doi. org/10.1097/00002030-200309050-00006 (2003).

16. Van Asten, L. et al. Pre-seroconversion immune status predicts the rate of CD4 T cell decline following HIV infection. AIDS https:// doi.org/10.1097/00002030-200409240-00004 (2004).

17. Karim, Q. A. et al. Effectiveness and safety of tenofovir gel, an antiretroviral microbicide, for the prevention of HIV infection in women. Science (80-.) https://doi.org/10.1126/science.1193748 (2010).

18. van Loggerenberg, F. et al. Establishing a cohort at high risk of HIV infection in South Africa: Challenges and experiences of the CAPRISA 002 acute infection study. PLoS ONE https://doi.org/10.1371/journal.pone.0001954 (2008).

19. Mlisana, K. et al. Rapid disease progression in HIV-1 subtype c-infected South African women. Clin. Infect. Dis. https://doi.org/ $10.1093 /$ cid/ciu573 (2014).

20. Paiardini, M. \& Müller-Trutwin, M. HIV-associated chronic immune activation. Immunol. Rev. https://doi.org/10.1111/imr.12079 (2013).

21. Cruikshank, W. \& Little, F. Interleukin-16: The ins and outs of regulating T-cell activation. Crit. Rev. Immunol. (2008).

22. Amiel, C. et al. Interleukin-16 (IL-16) inhibits human immunodeficiency virus replication in cells from infected subjects, and serum IL-16 levels drop with disease progression. J. Infect. Dis. https://doi.org/10.1086/314550 (1999).

23. Baier, M., Werner, A., Bannert, N., Metzner, K. \& Kurth, R. HIV suppression by interleukin-16. Nature https://doi.org/10.1038/ 378563a0 (1995).

24. Zhou, P., Goldstein, S., Devadas, K., Tewari, D. \& Notkins, A. L. Human CD4+ cells transfected with IL-16 cDNA are resistant to HIV-1 infection: Inhibition of mRNA expression. Nat. Med. https://doi.org/10.1038/nm0697-659 (1997).

25. Maciaszek, J. W. et al. IL-16 represses HIV-1 promoter activity. J. Immunol. 158, 5-8 (1997).

26. Gilpin, S. E. et al. Bone marrow-derived progenitor cells in end-stage lung disease patients. BMC Pulm. Med. https://doi.org/10. 1186/1471-2466-13-48 (2013).

27. Gee, K., Guzzo, C., Mat, N. F. C., Ma, W. \& Kumar, A. The IL-12 family of cytokines in infection, inflammation and autoimmune disorders. Inflamm. Allergy Drug Targets https://doi.org/10.2174/187152809787582507 (2009). 
28. Byrnes, A. A. et al. Immune activation and IL-12 production during acute/early HIV infection in the absence and presence of highly active, antiretroviral therapy. J. Leukoc. Biol. https://doi.org/10.1189/jlb.0708438 (2008).

29. Van Damme, J., Proost, P., Lenaerts, J. P. \& Opdenakker, G. Structural and functional identification of two human, tumor-derived monocyte chemotactic proteins (MCP-2 and MCP-3) belonging to the chemokine family. J. Exp. Med. https://doi.org/10.1084/ jem.176.1.59 (1992).

30. Cooper, A. M. \& Khader, S. A. IL-12p40: an inherently agonistic cytokine. Trends Immunol. https://doi.org/10.1016/j.it.2006.11. $002(2007)$.

31. Ha, S. J. et al. A novel function of IL-12p40 as a chemotactic molecule for macrophages. J. Immunol. 163, 2902-2908 (1999).

32. Hyrcza, M. D. et al. Distinct transcriptional profiles in ex vivo CD4+ and CD8+ T cells are established early in human immunodeficiency virus type 1 infection and are characterized by a chronic interferon response as well as extensive transcriptional changes in CD8+ T cells. J. Virol. https://doi.org/10.1128/jvi.01552-06 (2007).

33. Parrish, N. F. et al. Phenotypic properties of transmitted founder HIV-1. Proc. Natl. Acad. Sci. U. S. A. https://doi.org/10.1073/ pnas.1304288110 (2013).

34. Liao, W., Lin, J. X. \& Leonard, W. J. IL-2 family cytokines: New insights into the complex roles of IL-2 as a broad regulator of T helper cell differentiation. Curr. Opin. Immunol. https://doi.org/10.1016/j.coi.2011.08.003 (2011).

35. Collins, D. R., Gaiha, G. D. \& Walker, B. D. CD8+ T cells in HIV control, cure and prevention. Nat. Rev. Immunol. https://doi.org/ 10.1038/s41577-020-0274-9 (2020).

36. Lu, W. et al. $\mathrm{CD} 4: \mathrm{CD} 8$ ratio as a frontier marker for clinical outcome, immune dysfunction and viral reservoir size in virologically suppressed HIV-positive patients. J. Int. AIDS Soc. https://doi.org/10.7448/IAS.18.1.20052 (2015).

37. Sauce, D. et al. HIV disease progression despite suppression of viral replication is associated with exhaustion of lymphopoiesis. Blood https://doi.org/10.1182/blood-2011-01-331306 (2011).

38. Stacey, A. R. et al. Induction of a striking systemic cytokine cascade prior to peak viremia in acute human immunodeficiency virus type 1 infection, in contrast to more modest and delayed responses in acute hepatitis B and C virus infections. J. Virol. https://doi. org/10.1128/jvi.01844-08 (2009).

39. Brooks, D. G., Walsh, K. B., Elsaesser, H. \& Oldstone, M. B. A. IL-10 directly suppresses CD4 but not CD8 T cell effector and memory responses following acute viral infection. Proc. Natl. Acad. Sci. U. S. A. https://doi.org/10.1073/pnas.0914500107 (2010).

40. Liu, J. et al. IL-10-producing B cells are induced early in HIV-1 infection and suppress HIV-1-specific T cell responses. PLoS ONE https://doi.org/10.1371/journal.pone.0089236 (2014).

41. Dinarello, C. A. \& Thompson, R. C. Blocking IL-1: Interleukin 1 receptor antagonist in vivo and in vitro. Immunol. Today https:// doi.org/10.1016/0167-5699(91)90142-G (1991).

42. Carr, M. W., Roth, S. J., Luther, E., Rose, S. S. \& Springer, T. A. Monocyte chemoattractant protein 1 acts as a T-lymphocyte chemoattractant. Proc. Natl. Acad. Sci. U. S. A. https://doi.org/10.1073/pnas.91.9.3652 (1994).

43. Gu, L. et al. Control of $\mathrm{T}(\mathrm{H}) 2$ polarization by the chemokine monocyte chemoattractant protein-1. Nature https://doi.org/10. $1038 / 35006097$ (2000)

44. Patrikar, S. et al. Rate of decline in CD4 count in HIV patients not on antiretroviral therapy. Med. J. Armed Forces India https:// doi.org/10.1016/j.mjafi.2013.08.005 (2014).

\section{Acknowledgements}

We thank all the study participants, clinic and laboratory staff that participated in the CAPRISA 004 and 002 studies in Durban South Africa. We would additionally like to thank Dr. Nonhlanhla Yende-Zuma for her advice with the statistical analysis. Part of this project was funded by NIH R21 AI115978-01. This research and SN1 were supported by the National Research Foundation (NRF, Grant \#: 96354). Any opinion, finding, and conclusion or recommendations expressed in this material is that of the authors and the NRF does not accept liability in this regard. AS is supported by European \& Developing Countries Clinical Trials partnership (EDCTP) Career Development Fellowship (TMA2016CDF-1582). LRM is supported by CIHR New Investigator Award.

\section{Author contributions}

Designed the study: L.R.M., A.S.; Performed the experiments: S.N.1, A.S.; Analysed the data: S.N.1, A.S., R.P.M., F.O.; Wrote the first draft of the paper: S.N.1, A.S., L.R.M.; Supervised clinical and/or experimental aspects of the study: N.J.G., N.S., S.N.2, S.S.A.K., Q.A.K., L.R.M., A.S.; All authors contributed to the editing and finalisation of the manuscript.

\section{Competing interests}

The authors declare no competing interests.

\section{Additional information}

Supplementary Information The online version contains supplementary material available at https://doi.org/ 10.1038/s41598-022-06532-w.

Correspondence and requests for materials should be addressed to A.S.

Reprints and permissions information is available at www.nature.com/reprints.

Publisher's note Springer Nature remains neutral with regard to jurisdictional claims in published maps and institutional affiliations.

Open Access This article is licensed under a Creative Commons Attribution 4.0 International format, as long as you give appropriate credit to the original author(s) and the source, provide a link to the Creative Commons licence, and indicate if changes were made. The images or other third party material in this article are included in the article's Creative Commons licence, unless indicated otherwise in a credit line to the material. If material is not included in the article's Creative Commons licence and your intended use is not permitted by statutory regulation or exceeds the permitted use, you will need to obtain permission directly from the copyright holder. To view a copy of this licence, visit http://creativecommons.org/licenses/by/4.0/.

(c) The Author(s) 2022 